\section{Hinterbliebene Geschwister versterben häufig zu früh}

Kinder, die eine Schwester oder einen Bruder durch Tod verlieren, haben selbst ein erhöhtes Mortalitätsrisiko, so das Ergebnis einer dänischen Studie. Besonders hoch ist die Gefahr zwar im ersten Jahr nach dem Verlust der Schwester oder des Bruders, doch sie setzt sich noch lange fort.

$\mathrm{D}$ ass der Verlust eines nahen Verwandten das Mortalitätsrisiko Hinterbliebener steigert, ist bekannt. Wenig weiß man bislang allerdings darüber, wie sich der Tod eines Kindes auf dessen Geschwister auswirkt. Im Rahmen einer populationsbasierten Kohortenstudie sind Yongfu Yu und Kollegen von der Universität Aarhus dieser Frage jetzt mithilfe von Daten des schwedischen und dänischen Personenregisters nachgegangen (Dänemark: 1973-2009; Schweden: 19732008). In ihre Analyse schlossen sie über zwei Millionen dänische und knapp drei Millionen schwedische Kinder ab sechs Monaten mit mindes-

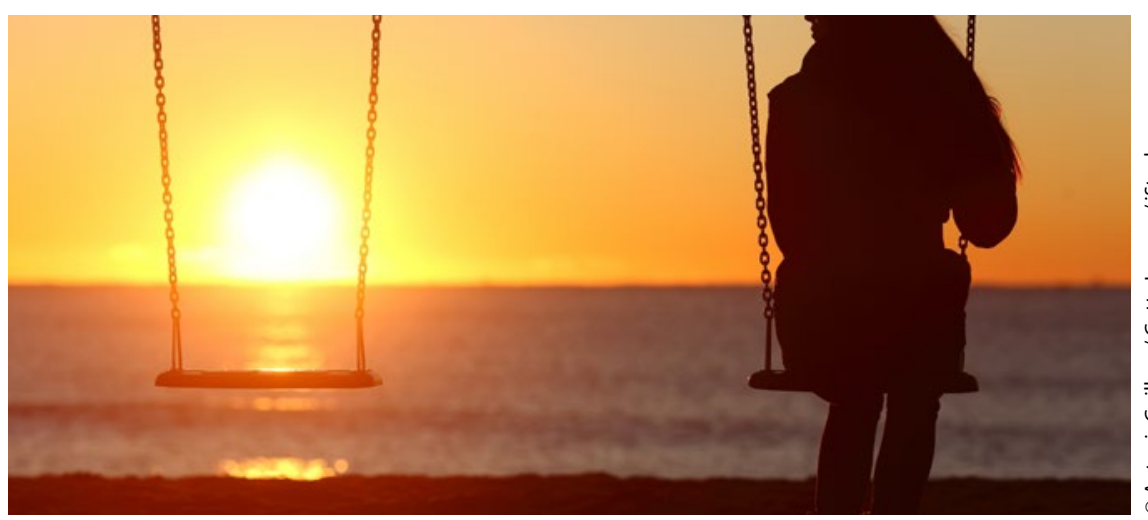

\section{Hitzewallungen als Warnsignal für Atherosklerose}

\section{Häufige Hitzewallungen bei relativ jungen Frauen beeinträchtigen offenbar nicht nur die Lebensqualität. Sie könnten zudem ein Indikator für ein erhöh- tes kardiovaskuläres Risiko sein.}

E ine Verbindung zwischen (peri-)me-
nopausalen Hitzewallungen und ei-
de schon in früheren Studien gesehen.
Die Untersuchungen haben allerdings Schwächen, u. a. weil die Häufigkeit von Hitzewallungen subjektiv und rückblickend ermittelt wurde oder weil andere kardiovaskuläre Risikofaktoren, etwa der sinkende Östradiolspiegel, nicht be- te (MRR 1,91). Als Beispiele nennen Yu und Kollegen Stoffwechselkrankheiten, Ernährungs- und Hormonstörungen, Krankheiten des Nerven- oder Kreislaufsystems sowie Suizid.

Das Sterberisiko des zurückgebliebenen Geschwisterkindes blieb über die gesamte Beobachtungszeit hinweg erhöht, unabhängig von der Art des Todes von Bruder oder Schwester oder davon, in welchem Alter der Hinterbliebene war, als diese verstarben. Allerdings war die Gefahr im ersten Jahr nach dem Verlust besonders hoch, vor allem dann, wenn das Geschwisterkind sich selbst das Leben genommen hatte. Während jedoch das Risiko eines Suizids für das zurückgebliebene Geschwisterkind nach diesem ersten Jahr mit der Zeit abfiel, hielt das erhöhte Sterberisiko infolge einer Krankheit nach dem einjährigen Gipfel weitaus länger an. Besonders hoch war das Mortalitätsrisiko generell für gleichgeschlechtliche Geschwister und für Geschwister, die altersmäßig nicht weit auseinander lagen. Die Wissenschaftler vermuten als Grund hierfür u.a. eine besonders enge Verbundenheit.

Nach dem Tod eines Kindes sollte den Geschwistern im Rahmen der Gesundheitsvorsorge besondere Beachtung geschenkt werden, fordern Yu und Kollegen, ganz besonders dann, wenn diese gleichgeschlechtlich oder in einem ähnlichen Alter seien. Sie sollten bei der Trauerbewältigung unterstützt und negative Effekte auf ihre Gesundheit so weit wie möglich reduziert werden. (cs)

Yu Y et al. Association of Mortality With the Death of a Sibling in Childhood. JAMA Pediatrics 2017; online 24. April 2017

rücksichtigt wurden. Ärzte der Universitäten Pittsburgh und Bern haben daher eine weitere Studie zum Thema aufgelegt, in der diese Fehler vermieden wurden. Den Ergebnissen zufolge sind häufige Hitzewallungen vor allem bei jüngeren Frauen ein kardiovaskulärer Risikoindikator: Die Betroffenen hatten eine signifikant schlechtere Endothelfunktion als Gleichaltrige ohne diese Beschwerden.

Die 272 Studienteilnehmerinnen im Alter zwischen 40 und 60 Jahren waren 